Document downloaded from:

http://hdl.handle.net/10251/45052

This paper must be cited as:

Araque Monrós, MC.; Vidaurre, A.; Gil Santos, L.; Gironés Bernabé, S.; Monleón Pradas, M.; Más Estellés, J. (2013). Study of degradation of a new PLA braided biomnaterial in buffer phosphate saline, basic and acid media, intended for the regeneration of tendons and ligaments. Polymer Degradation and Stability. 98(9):1563-1570.

doi:10.1016/j.polymdegradstab.2013.06.031.

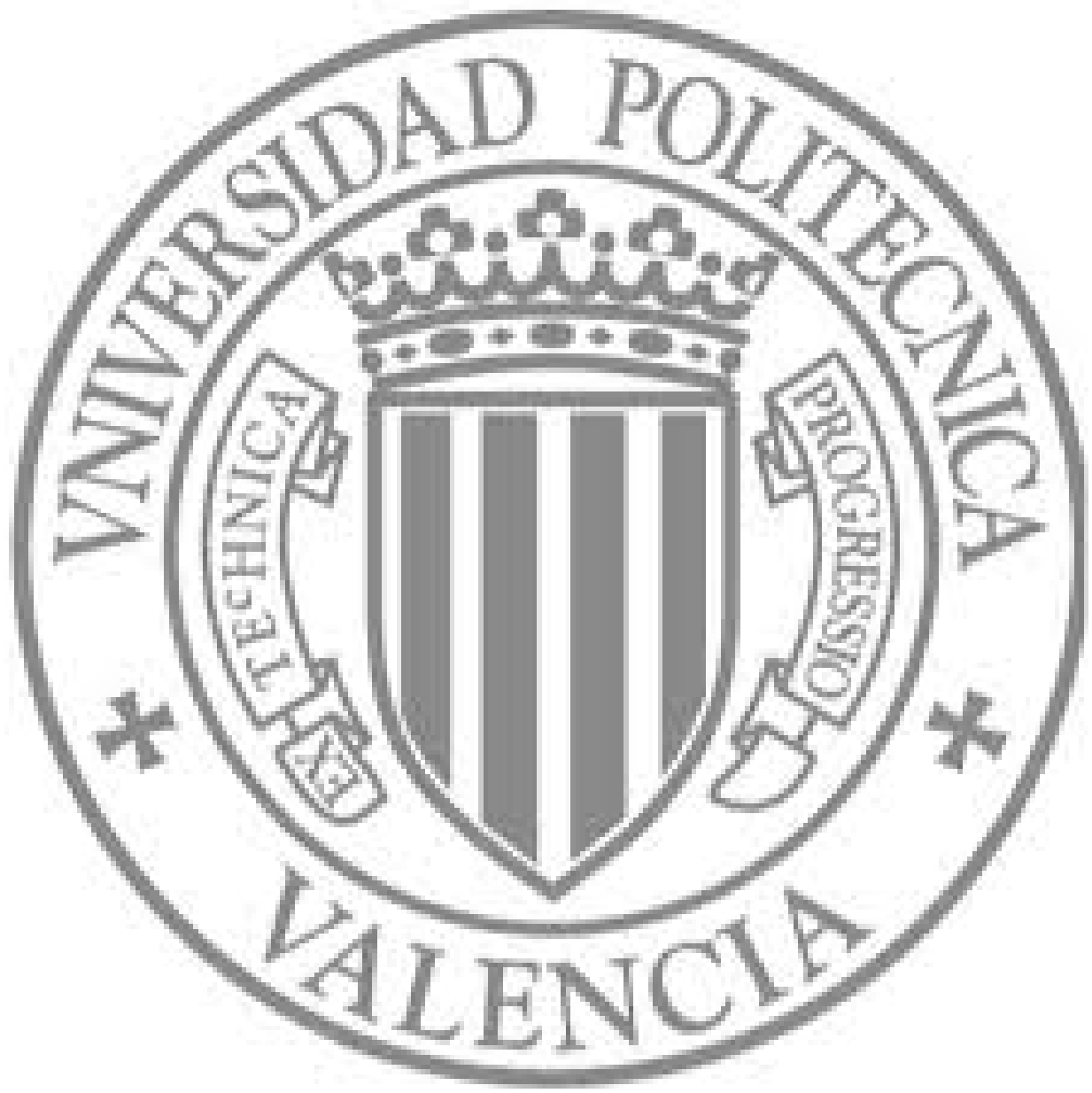

The final publication is available at

http://dx.doi.org/10.1016/j.polymdegradstab.2013.06.031

Copyright Elsevier 


\section{Study of the degradation of a new PLA braided biomaterial in buffer phosphate saline, basic and acid media, intended for the regeneration of tendons and ligaments.}

Araque-Monrós María C. ${ }^{\mathrm{a}, \mathrm{b}^{*}}$, Vidaurre Ana ${ }^{\mathrm{a}, \mathrm{b}}$, Gil-Santos Luis ${ }^{\mathrm{b}, \mathrm{c}, \mathrm{d}}$, Gironés Bernabé Sagrario $^{\mathrm{e}}$, Monleón-Pradas Manuel ${ }^{\mathrm{a}, \mathrm{b}}$, Más-Estellés Jorge ${ }^{\mathrm{b}}$.

${ }^{a}$ Centro de Biomateriales e Ingeniería Tisular, Universitat Politècnica de València, 46022, Valencia, Spain

${ }^{b}$ CIBER en Bioingeniería, Biomateriales y Nanomedicina, (CIBER-BBN), Valencia, Spainc

${ }^{c}$ Centro de Recuperación y Rehabilitación de Levante, (CRRL), Valencia, Spain 'Instituto Universitario de Investigación en Enfermedades Músculo-esqueléticas' Universidad Católica de Valencia (San Vicente Mártir). Spain eAsociación de Investigación de la Industria del Textil (AITEX), Alcoy, Alicante, Spain

*Corresponding author

Tel: 0034963877007 ext. 88939

Fax: 0034963877276

E-mail:marmon@upvnet.upv.es 


\begin{abstract}
The purpose of this study was to evaluate the effects of hydrolytic degradation on the properties of a PLA hollow braid designed as a new concept of biodegradable prosthesis for the regeneration of tendons and ligaments. The main function of the braided material is to bear mechanical loads while it is being replaced by the newly-generated tissue. The kinetics of braided material degradation is thus an important factor in determining the success of the product. In order to study this mechanism, PLA braid was subjected to a 12-month degradation process at $37^{\circ} \mathrm{C}$ in $\mathrm{PBS}$ at $\mathrm{pH} 7.4$ (to simulate the human physiological medium) and to accelerated degradation for one month in $\mathrm{pH} 12$ and $\mathrm{pH} 3$ solutions. Degradation of the braid subjected to hydrolysis was evaluated by weight loss, molecular weight distribution, mechanical properties, and calorimetric and morphologic analyses. The weight loss in a basic medium reached $21 \%$, versus no significant change in the other media. Average molecular weight was reduced by approximately $50 \%$ in the three media, with loss of mechanical properties in all cases. The morphological changes were more evident in the PLA degraded in the basic medium. The crystallinity of the material increased on first stages of degradation, regardless of the medium used.
\end{abstract}

Keywords: prosthesis, tendon, ligament, hydrolytic degradation 


\section{Introduction}

Polylactide (PLA) is an aliphatic polyester that can be obtained from natural sources like starch. Due to its chiral nature, there are two different PLA types: Poly(L-lactide) PLLA and Poly(D-lactide) PDLA; the former occurs naturally in abundance, and a small quantity of PDLA is normally added to it (1 - 10\%) in order to improve its processability.

PLA is biodegradable and bioabsorbable in a natural medium such as the human body and is widely used in many tissue-engineering applications. Due to its good mechanical properties it has been considered ideal for load-bearing applications such as fixation devices, sutures, etc. $[1,2]$. Due to the high strength of its fibers, it has also been tested as a replacement material for tendons and ligaments [3-5].

PLA is a hydrophobic material with a very slow degradation rate. It has been reported that high molecular-weight PLA can take between 2 and 5 years for total in vivo resorption [6]. The degradation rate depends on many factors, including degree of crystallinity, molecular weight, material morphology, etc. [7]. Many studies have been carried out on its hydrolytic degradation in different media, including: a neutral solution of buffer phosphate (PBS) at $37^{\circ} \mathrm{C}$ or higher to accelerate degradation [8-11], an alkaline solution at different temperatures [11-15], an acid solution [16, 17] and even studies on enzymatic degradation using enzymes as proteinase $\mathrm{K}[18,19]$.

The PLA hydrolysis mechanism is initiated by breaking the ester link of the polymeric chain when reacting with water. Degradation mainly begins in the amorphous regions of the material, where the macromolecule segments are more flexible and easily attacked by water. Degradation of these segments in the amorphous regions reduces molecular entanglements and increases chain mobility, which can then be reorganized. Once the amorphous regions have been attacked, degradation begins in the crystalline regions of 
the material, somewhat less susceptible to attack due to their ordered structure $[11,20]$. The mechanism associated with the hydrolysis of the ester group in a neutral or acid medium is different from that in an alkaline medium. In neutral or acid media, hydrolysis begins by protonation and is followed by the addition of water and the breaking of the ester link. In alkaline media the hydroxyl ions are joined to the carbons of the carbonyl group, after which the ester links are broken. This hydrolytic degradation of PLA has been reported to occur in the form of two mechanisms: surface erosion, in which degradation occurs only at the polymer-water interface, or bulk erosion, in which degradation is uniform throughout the polymer [21, 22]. These degradation mechanisms have a crucial impact on the success of an implant. PLA degradation products can affect cellular growth, tissue regeneration and the response of the organism. However, it has been reported that the low molecular-weight molecules produced by the fragmentation of polymer chains can be eliminated from the human body through the usual metabolic pathways without causing inflammatory responses $[21,23]$.

Our group is at present working on the production of a "regenerative prosthesis" for tendons and ligaments [24] with the aim of combining the load-bearing and replacement functions into a single construct, usually covered by a traditional prosthesis, with the ability to both regenerate lost tissue and be progressively replaced by it. This hypothesis combines a resistant PLA braid with a hollow interior that contains a progenitor cell supply. The cells are thus protected and kept in place more efficiently than when seeding takes place on top of the construct. The cell supply is from microparticles acting as cell carriers and as a three-dimensional scaffold. Finally, in order to avoid undesired tissue adherence, the exterior of the braid is coated with an impermeable non-adherent hyaluronic acid film, which allows the medium to move easily from inside to outside of 
the prosthesis or vice versa. The time taken by the seeded cells to regenerate a tendon or ligament can reach several months or even more than one year, and the prosthesis must preserve its mechanical properties along this term, in order to maintain the functionality of tissue. Once the natural tissue reaches its functionality, the prosthesis should gradually degrade until its complete disappearance. All the construct components are biodegradable and should be completely resorbed in the course of time. Changes in mechanical properties of prosthesis and the time taken for the degradation of the PLA braid of this prosthesis are of crucial importance and must be monitorized along the degradation process. The braid dimensions and characteristics (number of threads, braiding angle) can be adjusted to adapt the prosthesis to specific tendons or ligaments, Features and size of a specific braid have been given in a previous paper [25].

This paper describes a study of the evolution of the properties of this specific braided material throughout the hydrolysis degradation process at $37^{\circ} \mathrm{C}$ in different media, including: PBS with $\mathrm{pH} 7.4$, to simulate human physiological conditions, a solution of $\mathrm{NaOH}$ with $\mathrm{pH} 12$ and a solution of $\mathrm{HCl}$ with $\mathrm{pH} 3$ to accelerate the degradation process and compare the material's different degradation mechanisms. Changes in the physical and mechanical properties throughout degradation in the different media were measured by mass loss, differential scanning calorimetry (DSC), gel permeation chromatography (GPC), stress-strain tests and scanning electronic microscopy (SEM). 


\section{Materials and methods}

\subsection{Materials}

PLA microfibers (Ingeo, Natureworks PLA Polymer 6251D), with a linear density of 0.22 tex $(1$ tex=1 g/1000 m), made from poly-L-lactide PLLA, blended with $1.4 \%$ of poly-D-lactide PDLA, were used to manufacture a hollow cylindrical (tubular) braid using a Herzog NG 2/12-120 braiding machine (Oldenburg, Germany). The braid was made with 12 threads, each with 4 sets of 150 microfibers, giving a total of 7200 microfibers and a linear density of 1612.8 tex. The microfibers were braided around a PLA thread of 330 tex, subsequently removed to obtain the tubular braid. The effective cross section of the braid was computed from its linear mass densities (M/L), given in tex, through the equation (1):

$$
S=\frac{V}{L}=\frac{M}{L \rho_{P L A}}(1)
$$

PLA density was obtained from the manufacturer's data sheet as $\rho_{\mathrm{PLA}}=1.24 \mathrm{~g} / \mathrm{ml}$.

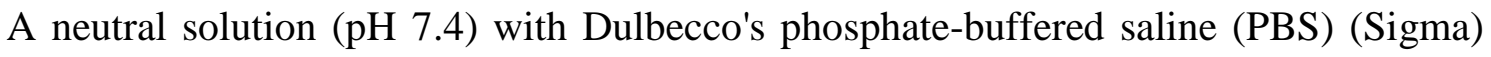
was prepared adding $0.2 \mathrm{mg} / \mathrm{ml}$ of sodium azide $99 \%$ (Aldrich). A solution of $\mathrm{NaOH}$ (Scharlab) $0.01 \mathrm{~N}$ with $\mathrm{pH} 12$ and another $\mathrm{HCl} 37 \%$ solution (Sigma) $0.001 \mathrm{~N}$ with $\mathrm{pH} 3$ were also prepared.

\subsection{PLA braid degradation}

Hydrolytic degradation of hollow braid PLA at $37^{\circ} \mathrm{C}$ was carried out in three different media: a solution of phosphate buffer saline (PBS) with $\mathrm{pH} 7.4$, a solution of $\mathrm{NaOH}$ $0.01 \mathrm{~N}$ with $\mathrm{pH} 12$ and a solution of $\mathrm{HCl} 0.001 \mathrm{~N}$ with $\mathrm{pH}$ 3. The test in a neutral medium had a duration of 12 months and those in acid and basic media were a month long. Three samples were prepared for each time and medium: braids $10 \mathrm{~cm}$ in length 
for mechanical testing and others $1 \mathrm{~cm}$ in length for the other tests. Before the test was carried out, each sample was weighed to obtain its initial mass $\left(m_{0}\right)$ using an XS Excellence Analytical Balance (USA). The PBS solution was renewed weekly and acid and basic solutions every $48 \mathrm{~h}$. Solution $\mathrm{pH}$ was measured before each change of medium using a pH-meter (Eutech Instruments Model PH1500, Singapore) in order to verify that solution $\mathrm{pH}$ remained stable. The samples were regularly taken out of the degradation media, washed with distilled water at $50^{\circ} \mathrm{C}$ (so as not to affect the properties of the material and to remove any salt that could have been deposited on its surface) and dried at $37^{\circ} \mathrm{C}$ in a vacuum until constant weight before being tested. The braid mass after degradation $\left(m_{d}\right)$ enables mass loss to be calculated through the equation (2):

Mass $\operatorname{loss} \%=\frac{\left(m_{0}-m_{d}\right)}{m_{0}} \times 100$

\subsection{Morphology}

Non-degraded and degraded PLA braid morphology were evaluated using a scanning electronic microscope (SEM) (JEOL JSM-5410) working at $10 \mathrm{kV}$, before which the samples were coated with a thin gold layer using the sputtering technique.

\subsection{Molecular weight}

To determine the distribution of molecular weights, gel permeation chromatography (GPC) was used on a Chromatograph HPLC/GPC, (Waters Model 1525, US) with a Refractive Index Detector. Samples were solved in tetrahydrofuran; grade ACS (THF) (Sharlab) $6 \mathrm{mg} / \mathrm{ml}$. Polystyrene standards were used for calibration. From the obtained distribution of molecular weights, the following were computed: average molecular 
weight in weight $(\mathrm{Mw})$, average molecular weight in number $\left(\mathrm{M}_{\mathrm{n}}\right)$ and polydispersity index (PDI) obtained as the $\mathrm{Mw} / \mathrm{Mn}$ rate.

\subsection{Thermal analysis}

PLA braided material thermal properties before and after the degradation process were measured with a calorimeter (METTLER TOLEDO, Model DSC 823e, Switzerland) using nitrogen as purge gas with a flow of $20 \mathrm{ml} / \mathrm{min}$ and aluminum pans of $40 \mu \mathrm{l}$. The temperature of the equipment was calibrated with Indium, and the melting enthalpy of Indium was used to calibrate the heat flux. One heating scan from $0^{\circ} \mathrm{C}$ to $210^{\circ} \mathrm{C}$ at $10^{\circ} \mathrm{C} / \mathrm{min}$ was evaluated. The weight of the tested sample ranged between 5 and $10 \mathrm{mg}$. Different parameters were determined from the thermogram: glass transition temperature, $\mathrm{T}_{\mathrm{g}}$, melting temperature, $\mathrm{T}_{\mathrm{m}}$, melting crystallization enthalpy, $\Delta \mathrm{H}_{\mathrm{c}}$ and melting enthalpy, $\Delta \mathrm{H}_{\mathrm{m}}$. The crystallinity of the PLA braided material, $X_{c}$, was determined according to equation (3), making use of the reported melting enthalpy of PLA crystals, $\Delta H^{o}{ }_{m}=93.7 \mathrm{~J} / \mathrm{g}[26]$.

$$
X_{c}=\frac{\Delta H_{m}}{\Delta H_{m}^{o}} \times 100
$$

\subsection{Tensile properties}

Stress-strain tests of PLA braided material before and after different degradation times in different media were carried out in a hydraulic device (MICROTEST SCM 400098 , Spain) at $5 \mathrm{~mm} / \mathrm{min}$, using a $400 \mathrm{~N}$ load cell and recording 1 data/s. Measurements were performed in triplicate at room temperature.

The Young's modulus of the braided material was taken as the slope of the regression line in the linear region of the stress-strain curve. The limit of the "toe" region of the 
deformation curve was defined as the deformation at which the linear region of the curve begins. The samples were subjected to stress-strain assays until failure and the breaking load and the elongation (\%) at break were determined.

\subsection{Statistical Analysis}

The statistical analysis was carried out using the Student t-test with SPSS 16.0 software and a statistical significance value of $\mathrm{p}<0.05$. 


\section{Results}

\subsection{Weight loss}

The results of the weight loss of the braided PLA material degraded in several media as a function of time are given in Fig. 1 and show that the samples treated in the basic medium had a $21 \%$ weight loss after 30 days (Fig. 1a). The samples treated in acid and PBS medium showed a slight weight loss $(0.65 \%$ after 30 days in acid medium and less than $1 \%$ after a year in PBS). The statistical analysis by the Student's $t$ test shows that the samples treated in the basic medium had a significant weight loss after the seventh day of testing, while those treated in the acid medium showed no significant weight loss for the duration of the study. The braids treated in the PBS medium had a significant, although negligible weight loss the first two months (Fig. 1b). From then on, no statistically significant weight was observed. No changes were observed in the $\mathrm{pH}$ of any media. 
a)

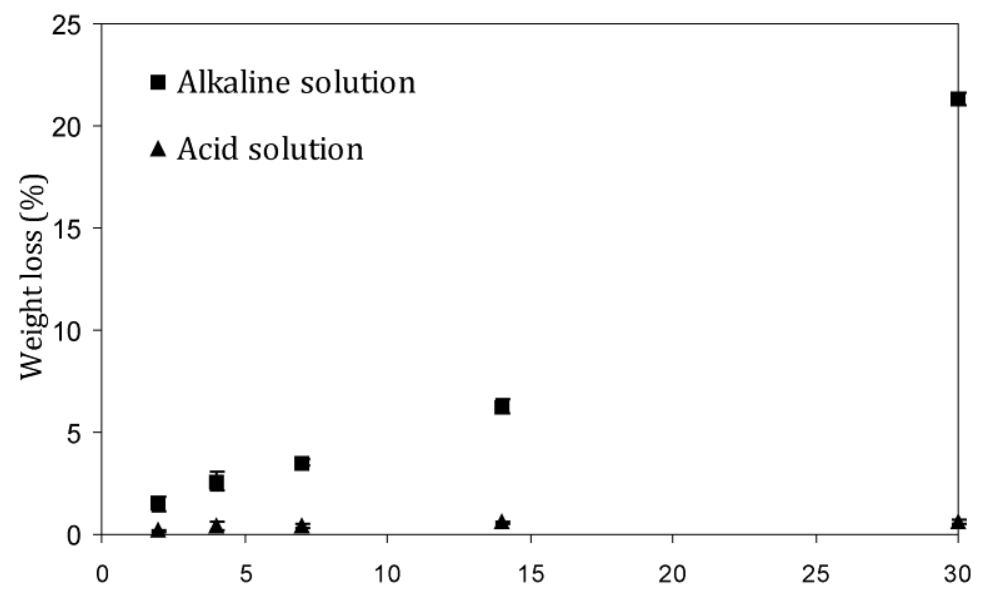

b)

Time (days)

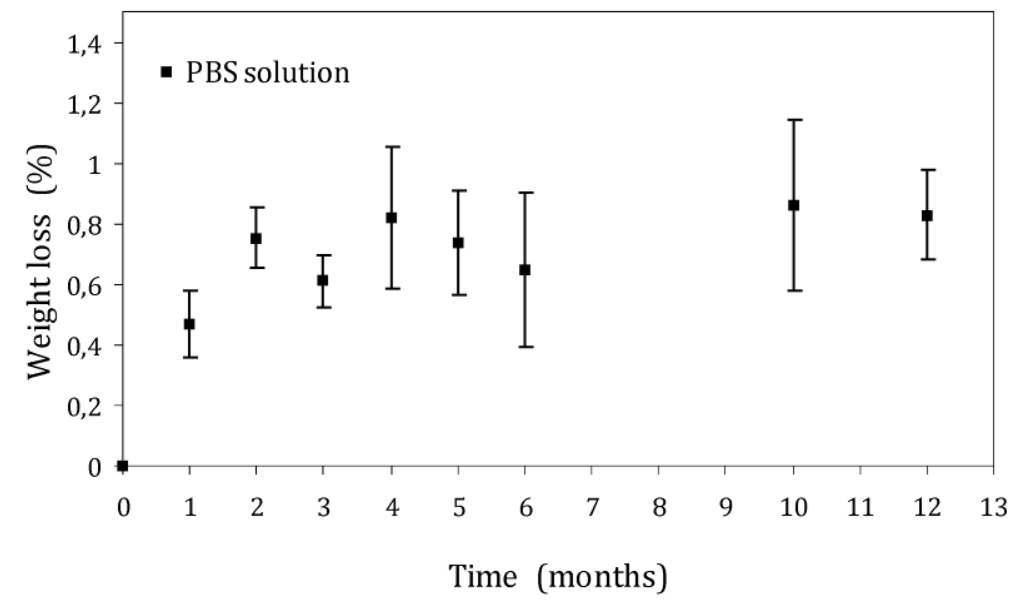

Fig. 1 Weight loss of PLA braided material as a function of degradation time in different media. a) Samples immersed in basic and acid media at $37^{\circ} \mathrm{C}$ for one month; b) samples immersed in $\mathrm{PBS}$ at $37^{\circ} \mathrm{C}$ for one year.

\subsection{Braid morphology}

SEM micrographs of PLA braided after 6 months degradation in PBS are shown in Fig. 2. The surface of the braid before degradation $\left(T_{0}\right)$ looks smooth, and after 4 and 6 months the fibers took on a rougher appearance $\left(\mathrm{T}_{0}\right)$.

The morphology of the PLA braided before and after 15 and 30 days of degradation in basic and acid media can be seen in Fig. 3. After 15 days, the braids in the basic medium show the early cracks on the surface of the microfibers, while no imperfections 
can be observed in those treated in the acid medium. After 30 days there were notable changes in the surface of the braided material degraded in the basic medium, with a considerable number of perforations on the surface of the microfibers. The microfibers of the samples treated in the acid medium showed transversal irregularities and cracks.
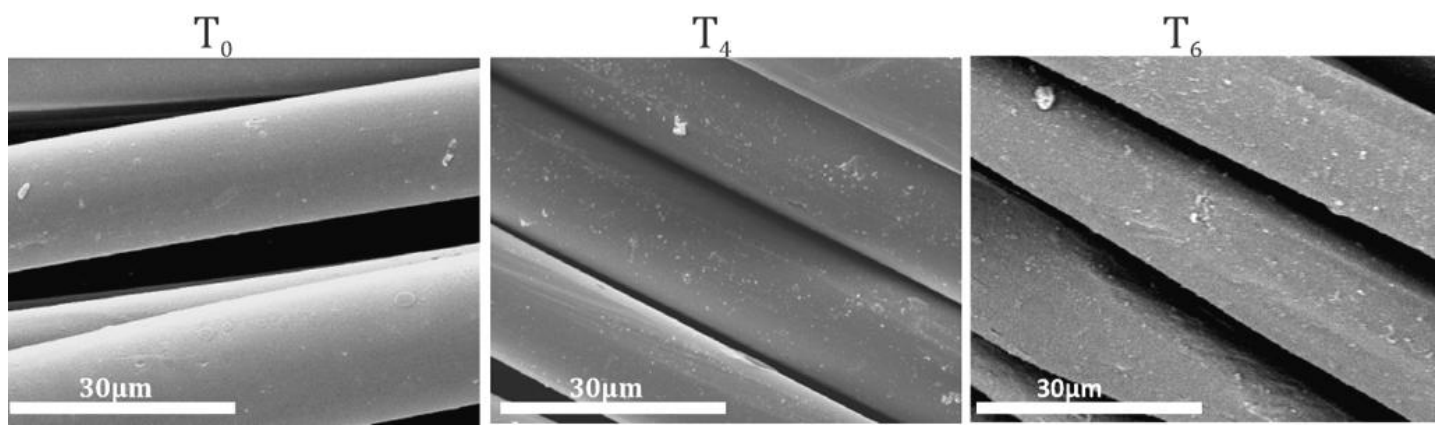

Fig. 2 SEM micrographs of PLA braid before and after degradation in PBS for different times. $\mathrm{T}_{0}$, non-degraded sample, $\mathrm{T}_{4}$ after 4 months and $\mathrm{T}_{6}$ after 6 months' degradation.
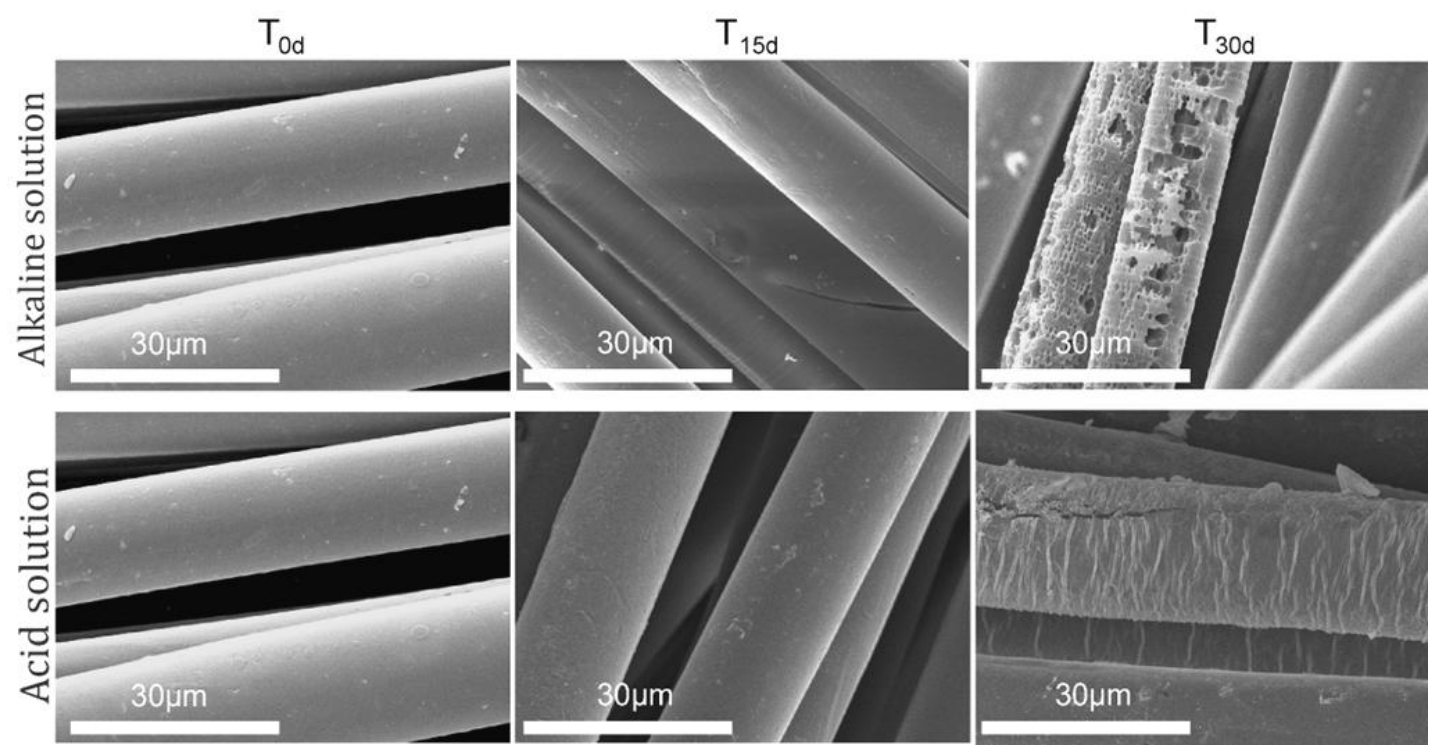

Fig. 3 SEM micrographs of PLA braid before and after degradation in alkaline and acid media for different times. $\mathrm{T}_{0}$, non-degraded sample, $\mathrm{T}_{15 \mathrm{~d}}$ after 15 days, and $\mathrm{T}_{30 \mathrm{~d}}$ after 30 days' degradation.

\subsection{Molecular weight}


The distribution of the molecular weights of the PLA braid before and after degradation by hydrolysis in PBS, alkaline and acid media are shown in Fig. 4. When degradation is in progress the molecular weights have lower Mw values and the base of the molecular weight distribution curve becomes wider, indicating an increase of the low molecularweight chains.

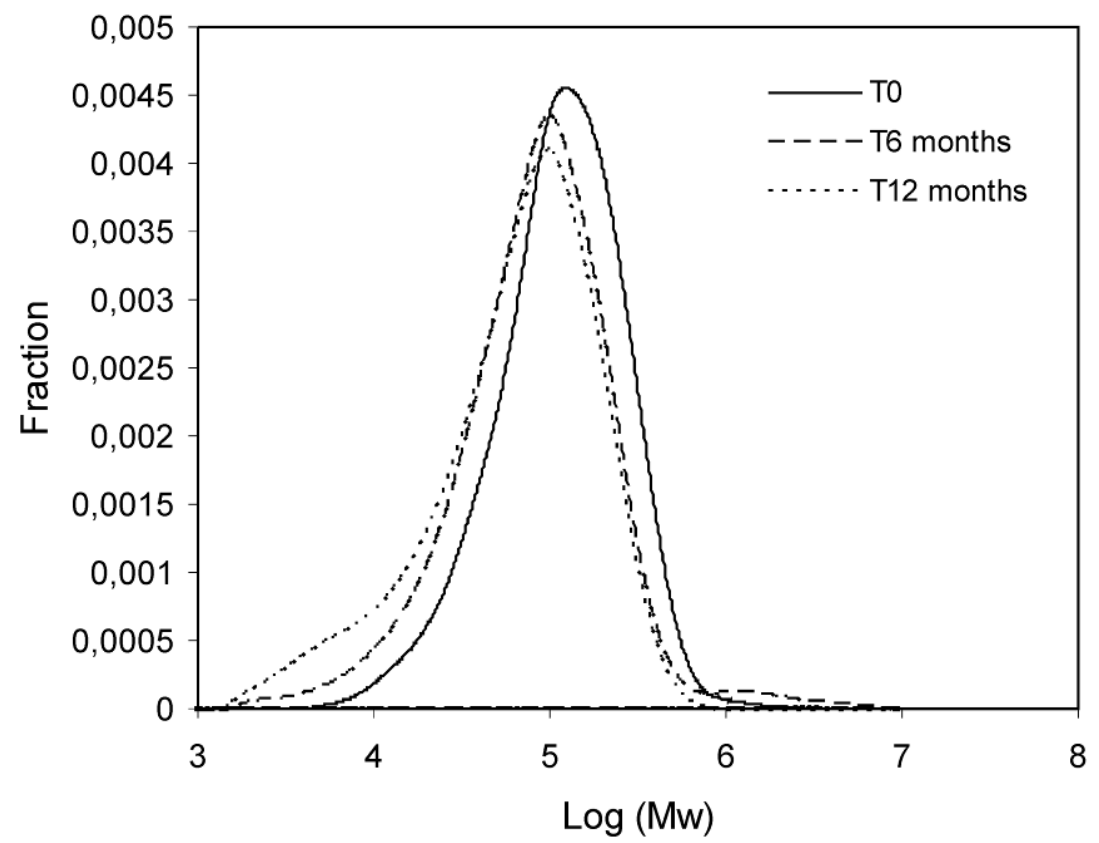

Fig. 4 GPC curves of PLA braid before and after PBS degradation for different time periods. $\mathrm{T}_{0}$, non-degraded sample, $\mathrm{T}_{6}$ after 6 months, and $\mathrm{T}_{12}$ after 12 months' degradation.

The molecular weights of PLA braid degraded for different periods in PBS are shown in Table 1. Applying the statistical Student's t test with $\mathrm{p}<0.05$ no significant change was observed in $\mathrm{Mw}$ until the eighth month of degradation. However, significant changes in Mn were observed after the third month. Average Mw is $50 \%$ of the initial value and average $\mathrm{Mn}$ is $60 \%$ of the initial value after 12 months' degradation. PDI increased throughout the test. 


\begin{tabular}{|c|c|c|c|}
\hline Time (months) & Mw $\left(\right.$ gmole $\left.^{-1}\right)$ & Mn $\left(\right.$ gmole $\left.^{-1}\right)$ & PDI \\
\hline 0 & $(1.6 \pm 0.02) \times 10^{5}$ & $(7.7 \pm 0.5) \times 10^{4}$ & $2.1 \pm 0.16$ \\
\hline 1 & $(1.4 \pm 0.01) \times 10^{5}$ & $(5.9 \pm 0.8) \times 10^{4}$ & $2.4 \pm 0.34$ \\
\hline 3 & $(1.2 \pm 0.07) \times 10^{5}$ & $(5.3 \pm 1.2) \times 10^{4}$ & $2.3 \pm 0.39$ \\
\hline 5 & $(1.2 \pm 0.04) \times 10^{5}$ & $(5.8 \pm 0.2) \times 10^{4}$ & $2.2 \pm 0.16$ \\
\hline 6 & $(1.3 \pm 0.3) \times 10^{5}$ & $(3.9 \pm 1.1) \times 10^{4}$ & $3.3 \pm 0.18$ \\
\hline 8 & $(7.4 \pm 1.3) \times 10^{4}$ & $(3.2 \pm 0.7) \times 10^{4}$ & $2.4 \pm 0.14$ \\
\hline 10 & $(7.9 \pm 0.7) \times 10^{4}$ & $(3.1 \pm 0.9) \times 10^{4}$ & $2.5 \pm 0.03$ \\
\hline 12 & $(7.9 \pm 0.3) \times 10^{4}$ & $(3.1 \pm 0.2) \times 10^{4}$ & $2.5 \pm 0.22$ \\
\hline
\end{tabular}

Table 1 Molecular weight average in weight $(\mathrm{Mw})$, number $(\mathrm{Mn})$ and polydispersity index (PDI) of PLA braid before and after different degradation times in PBS at $37^{\circ} \mathrm{C}$. Data measured with GPC.

The data obtained for the distribution of molecular weights using GPC in basic and acid media are shown in Table 2. The Mw of the braids degraded in both media significantly decreased from the second day, as was shown by the statistical analysis ( $t$ Student with $\mathrm{p}<0.05)$; the same behavior was observed for Mn. Non-significant changes were observed in the PDI throughout the degradation period in both media.

\begin{tabular}{|c|c|c|c|c|c|c|}
\hline \multirow{2}{*}{$\begin{array}{c}\text { Degradation } \\
\text { time (days) }\end{array}$} & \multicolumn{4}{|c}{ Alkaline solution } & \multicolumn{3}{c|}{ Acid solution } \\
\cline { 2 - 7 } & Mw $\left(\mathbf{g ~ m o l e}^{-1}\right)$ & Mn $\left(\mathrm{g} \mathrm{mole}^{-1}\right)$ & PDI & Mw $\left(\mathrm{g} \mathrm{mole}^{-1}\right)$ & Mn $\left(\mathrm{g} \mathrm{mole}^{-1}\right)$ & PDI \\
\hline 0 & $(1.61 \pm 0.02) \times 10^{5}$ & $(7.7 \pm 0.5) \times 10^{4}$ & $2.09 \pm 0.16$ & $(1.61 \pm 0.02) \times 10^{5}$ & $(7.7 \pm 0.5) \times 10^{4}$ & $2.10 \pm 0.16$ \\
\hline 2 & $(1.33 \pm 0.04) \times 10^{5}$ & $(6.8 \pm 0.4) \times 10^{4}$ & $1.96 \pm 0.06$ & $(1.27 \pm 0.06) \times 10^{5}$ & $(5.5 \pm 0.5) \times 10^{4}$ & $2.18 \pm 0.23$ \\
\hline 4 & $(1.31 \pm 0.05) \times 10^{5}$ & $(6.4 \pm 1) \times 10^{4}$ & $2.01 \pm 0.24$ & $(1.32 \pm 0.01) \times 10^{5}$ & $(5.6 \pm 0.9) \times 10^{4}$ & $2.40 \pm 0.18$ \\
\hline 7 & $(1.3 \pm 0.05) \times 10^{5}$ & $(5.8 \pm 1.2) \times 10^{4}$ & $2.33 \pm 0.35$ & $(1.3 \pm 0.05) \times 10^{5}$ & $(5.9 \pm 1.4) \times 10^{4}$ & $2.21 \pm 0.2$ \\
\hline 14 & $(1.28 \pm 0.04) \times 10^{5}$ & $(6.3 \pm 0.8) \times 10^{4}$ & $2.05 \pm 0.22$ & $(1.25 \pm 0.04) \times 10^{5}$ & $(5.8 \pm 0.8) \times 10^{4}$ & $2.18 \pm 0.28$ \\
\hline 30 & $(1.26 \pm 0.02) \times 10^{5}$ & $(5.3 \pm 0.9) \times 10^{4}$ & $2.44 \pm 0.35$ & $(1.25 \pm 0.05) \times 10^{5}$ & $(5 \pm 0.8) \times 10^{4}$ & $2.52 \pm 0.2$ \\
\hline
\end{tabular}


Table 2 Molecular weight averages in weight $(\mathrm{Mw})$, in number $(\mathrm{Mn})$ and the polydispersity index (PDI) of PLA braid before and after different degradation times in alkaline and acid media at $37^{\circ} \mathrm{C}$. Data measured with GPC.

\subsection{Thermal analysis}

The DSC technique was used to find the thermal properties of the PLA braid before and after hydrolytic degradation at $37^{\circ} \mathrm{C}$. The $\mathrm{T}_{\mathrm{g}}, \Delta \mathrm{H}_{\mathrm{c}}, \Delta \mathrm{H}_{\mathrm{m}}, \mathrm{T}_{\mathrm{m}}$ and the $X_{\mathrm{c}}$ of PLA braids exposed to different degradation media were computed from the thermograms obtained. The results of the heating scan obtained from the braid degraded in PBS for 12 months are reported in Table 3. From these curves it was found that the $\Delta \mathrm{H}_{\mathrm{m}}, X_{\mathrm{c}}$ and $\mathrm{T}_{\mathrm{g}}$ of the material apparently increased on first stages of degradation time, while $T_{m}$ and $\Delta H_{c}$ decreased with time.

\begin{tabular}{|c|c|c|c|c|c|}
\hline \multirow{2}{*}{$\begin{array}{c}\text { Degradation } \\
\text { time } \\
\text { (months) }\end{array}$} & \multicolumn{5}{|c|}{ PBS solution } \\
\cline { 2 - 6 } & $\mathbf{T}_{\mathbf{g}}\left({ }^{\circ} \mathbf{C}\right)$ & $\Delta \mathbf{H}_{\mathbf{m}}\left(\mathbf{J g}^{-\mathbf{1}}\right)$ & $\Delta \mathbf{H}_{\mathbf{c}}\left(\mathbf{J g}^{-\mathbf{1}}\right)$ & $\mathbf{T}_{\mathbf{m}}\left({ }^{\circ} \mathbf{C}\right)$ & $\mathbf{X}_{\mathbf{c}}(\mathbf{\%})$ \\
\hline 0 & 62.4 & 43.8 & 10.7 & 167.6 & 36 \\
\hline 1 & 77.0 & 48.0 & 5.81 & 170.2 & 42.2 \\
\hline 3 & 76.8 & 48.0 & 5.66 & 168.0 & 42.3 \\
\hline 6 & 77.1 & 50.7 & 5.26 & 168.4 & 45.4 \\
\hline 8 & 76.8 & 48.5 & 5.56 & 164.7 & 42.9 \\
\hline 12 & 76.5 & 48.1 & 4.76 & 163.9 & 43.6 \\
\hline
\end{tabular}

Table 3 Thermal properties of PLA braid before and after degradation in PBS at $37^{\circ} \mathrm{C}$ for 12 months.

Table 4 shows the thermal properties of the PLA braided material degraded in basic and acid media. $\Delta \mathrm{H}_{\mathrm{m}}, X_{\mathrm{c}}$ and $\mathrm{T}_{\mathrm{g}}$ of the material increased on first stages of degradation time; $\mathrm{T}_{\mathrm{m}}$ does not show a clear trend. 


\begin{tabular}{|c|c|c|c|c|c|c|c|c|c|c|}
\hline \multirow[b]{2}{*}{$\begin{array}{l}\text { Degradation } \\
\text { time (days) }\end{array}$} & \multicolumn{5}{|c|}{ Alkaline solution } & \multicolumn{5}{|c|}{ Acid solution } \\
\hline & $\begin{array}{c}\mathbf{T}_{\mathbf{g}} \\
\left({ }^{\circ} \mathbf{C}\right)\end{array}$ & $\begin{array}{l}\Delta \mathbf{H}_{\mathrm{m}} \\
\left(\mathrm{Jg}^{-1}\right)\end{array}$ & $\begin{array}{l}\Delta \mathbf{H}_{\mathrm{c}} \\
\left(\mathrm{Jg}^{-1}\right)\end{array}$ & $\begin{array}{c}\mathbf{T}_{\mathbf{m}} \\
\left({ }^{\circ} \mathbf{C}\right)\end{array}$ & $\begin{array}{l}X_{c} \\
(\%) \\
\end{array}$ & $\begin{array}{c}\mathbf{T}_{\mathrm{g}} \\
\left({ }^{\circ} \mathbf{C}\right)\end{array}$ & $\begin{array}{c}\Delta \mathbf{H}_{\mathrm{m}} \\
\left(\mathrm{Jg}^{-1}\right) \\
\end{array}$ & $\begin{array}{l}\Delta \mathbf{H}_{\mathrm{c}} \\
\left(\mathrm{Jg}^{-1}\right)\end{array}$ & $\begin{array}{c}\mathbf{T}_{\mathbf{m}} \\
\left({ }^{\circ} \mathbf{C}\right) \\
\end{array}$ & $\begin{array}{c}X_{c} \\
(\%) \\
\end{array}$ \\
\hline 0 & 62.4 & 43.8 & 10.7 & 167.6 & 36 & 62.4 & 43.8 & 10.7 & 167.6 & 36 \\
\hline 2 & 69.0 & 46.6 & 7.45 & 164.6 & 39.2 & 75.5 & 47.1 & 7.46 & 167.2 & 39.6 \\
\hline 14 & 75.9 & 48.9 & 6.58 & 167.1 & 42.3 & 75.9 & 48.1 & 7.18 & 167.3 & 40.9 \\
\hline 30 & 77.1 & 47.9 & 5.28 & 167.3 & 42.6 & 77.1 & 47.3 & 6.23 & 164.9 & 41.1 \\
\hline
\end{tabular}

Table 4 Thermal properties of PLA braid before and after degradation in basic and acid media at $37^{\circ} \mathrm{C}$ for 1 month

\subsection{Tensile properties}

Fig 5 shows the results of the stress-strain tests conducted on the PLA braids degraded in different media. It can be observed that the braid subjected to PBS (Fig 5a) showed a remarkable decrease in Young's modulus after 2 months' degradation. The same tendency is observed in the braids subjected to basic and acid media after 15 days' degradation (Figs. 5b and 5c) and that the toe zone $\varepsilon_{\text {toe }}$ is longer in degraded than in non-degraded samples. The ranges were $\varepsilon_{\text {toe }} 3 \% \pm 0.1$ to $\varepsilon_{\text {toe }} 8 \% \pm 1.5$ in braids treated in PBS for 12 months, $\varepsilon_{\text {toe }} 10 \% \pm 0.1$ in samples degraded in the basic medium and $\varepsilon_{\text {toe }}$ $7 \% \pm 0.6$ in those degraded in the acid medium for a period of one month.

The Young's modulus, breaking load and elongation of PLA braids degraded in the different media were estimated by means of a stress-strain test. The data obtained are shown in Tables 5 and 6.

The data show that both the Young's modulus and the breaking load of the PLA braids decrease with degradation time, regardless of the medium, while elongation increases with degradation time in all cases. 

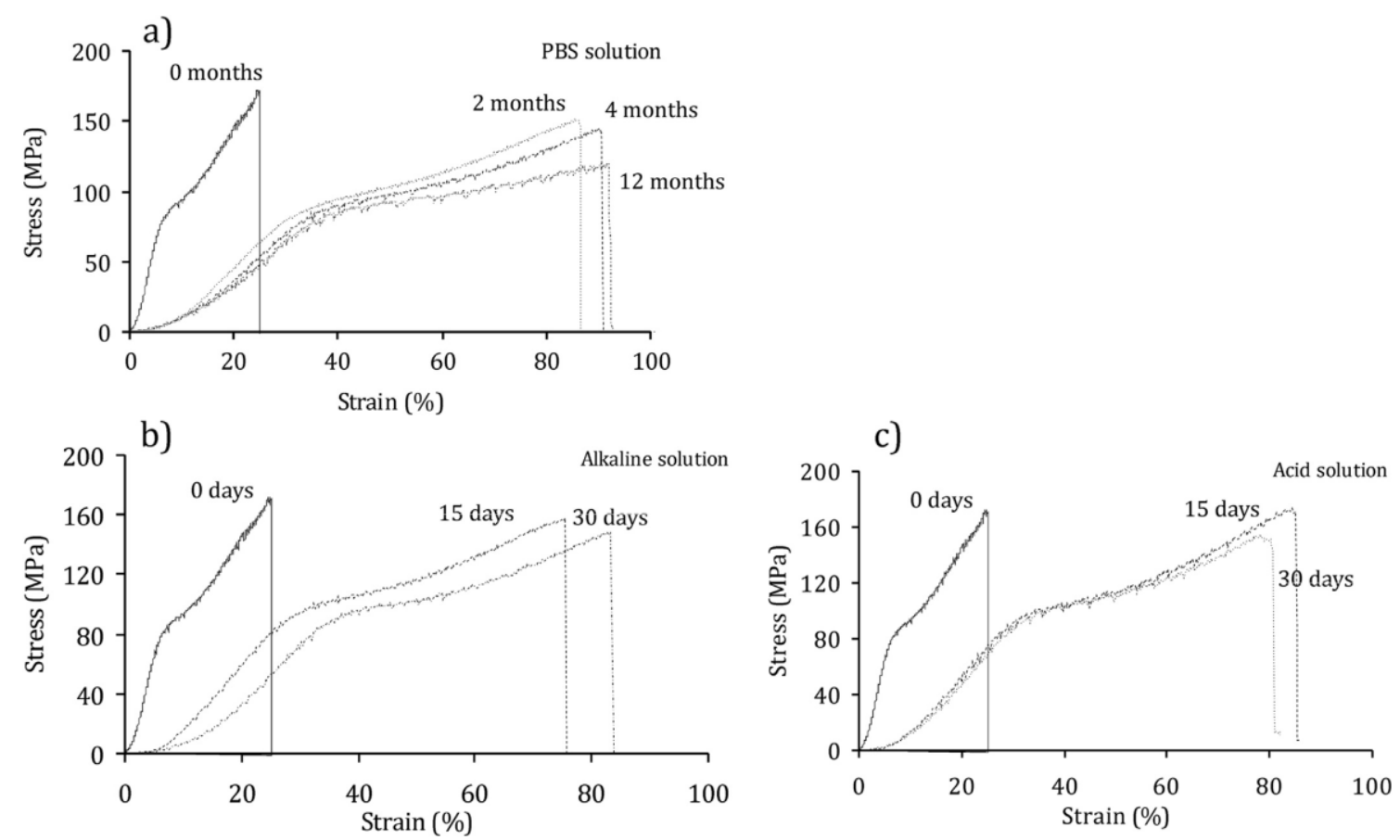

Fig.5 Stress-strain curves for the PLA braids before and after degradation at $37^{\circ} \mathrm{C}$ in a)

PBS, b) basic medium and c) acid medium

\begin{tabular}{|c|c|c|c|}
\hline $\begin{array}{c}\text { Degradation } \\
\text { time } \\
\text { (months) }\end{array}$ & $\begin{array}{c}\text { Young's } \\
\text { modulus } \\
\text { (MPa) }\end{array}$ & $\begin{array}{c}\text { Breaking } \\
\text { load } \\
\mathbf{( N )}\end{array}$ & $\begin{array}{c}\text { Elongation } \\
\mathbf{( \% )}\end{array}$ \\
\hline 0 & $1370 \pm 87$ & $217 \pm 0.1$ & $4 \pm 0.5$ \\
\hline 2 & $341 \pm 27$ & $190 \pm 8$ & $28 \pm 4$ \\
\hline 4 & $311 \pm 16$ & $186 \pm 6$ & $30 \pm 4$ \\
\hline 12 & $282 \pm 13$ & $152 \pm 5$ & $32 \pm 8$ \\
\hline
\end{tabular}

Table 5. Young's Modulus, breaking load and elongation of PLA braids before and after degradation in $\mathrm{PBS}$ at $37^{\circ} \mathrm{C}$ 


\begin{tabular}{|c|c|c|c|c|c|c|}
\hline \multirow[b]{2}{*}{$\begin{array}{c}\text { Degradation } \\
\text { time } \\
\text { (days) }\end{array}$} & \multicolumn{3}{|c|}{ Alkaline solution } & \multicolumn{3}{|c|}{ Acid solution } \\
\hline & $\begin{array}{c}\text { Young } \\
\text { modulus } \\
\text { (MPa) }\end{array}$ & $\begin{array}{c}\text { Breaking } \\
\text { load } \\
(\mathrm{N})\end{array}$ & $\begin{array}{c}\text { Elongation } \\
(\%)\end{array}$ & $\begin{array}{c}\text { Young } \\
\text { modulus } \\
(\mathrm{MPa})\end{array}$ & $\begin{array}{l}\text { Breaking } \\
\text { load } \\
\text { (N) }\end{array}$ & $\begin{array}{c}\text { Elongation } \\
(\%)\end{array}$ \\
\hline 0 & $1370 \pm 87$ & $217 \pm 0.1$ & $4 \pm 0.5$ & $1370 \pm 87$ & $217 \pm 0.1$ & $4 \pm 0.5$ \\
\hline 15 & $427 \pm 13$ & $211 \pm 15$ & $28 \pm 2$ & $403 \pm 15$ & $214 \pm 12$ & $28 \pm 1$ \\
\hline 30 & $392 \pm 17$ & $199 \pm 11$ & $33 \pm 2$ & $414 \pm 27$ & $202 \pm 8$ & $29 \pm 0.02$ \\
\hline
\end{tabular}

Table 6. Young's modulus, breaking load and elongation of PLA braids before and after degradation in basic and acid media.

\section{Discussion}

The objective of this study was to evaluate the evolution of mechanical properties and degradation process of this specific hollow PLA braid with degradation time. This prosthesis is different of any other appearing on the literature, and then must be checked in order to verify it's suitable for a tendon or ligament prosthesis. Hydrolytic degradation was performed in three different media: PBS with a $\mathrm{pH}$ of 7.4, simulating human physiological $\mathrm{pH}$, and solutions with extreme $\mathrm{pH}$ values of 12 and 3, to simulate accelerated degradation, so as to be able to predict the behavior of the material after degradation in extreme $\mathrm{pH}$ conditions or after longer degradation time in neutral $\mathrm{pH}$.

Weight loss depends on the $\mathrm{pH}$ of the degradation medium. While only $1 \%$ weight loss was measured after one year in PBS and one month in an acid medium, this figure was $21 \%$ in the basic medium. The remarkable difference is due to the mechanism associated with the hydrolysis of the ester group in basic medium produced by the hydroxyl ions attacking the carbon in the carbonyl. This hydroxyl ion has a strong catalytic effect that accelerates degradation and causes an increase of the hydroxyl ($\mathrm{OH})$ and carboxylic groups (-COOH), so that the samples become more hydrophilic and more susceptible to degradation. This affects sample morphology, as can be observed in 
the SEM micrographs. After 30 days of degradation in basic medium, the PLA braid fibers present holes, indicating superficial erosion in conjunction with bulk degradation. Yuan $[11,12]$ obtained a similar result for hydrolytic degradation of PLLA fibers. However no significant changes were observed in the morphology of PLA braids degraded in PBS or acid medium. As there were no changes in the sample weights, this suggests that the low molecular weight chains caused by the chain scission are either not water soluble or remain trapped inside the sample. Tsuji concluded that PLA degradation in basic medium is produced by superficial erosion accompanied by a considerable weight loss, while acid or PBS degradation are produced in bulk with very little weight loss $[14,16,27]$.

The scission of ester groups due to hydrolytic degradation produces low molecular weight chains and a consequent decrease in the sample $\mathrm{Mw}$ and $\mathrm{Mn}$ as degradation proceeds. The samples degraded in the three media presented a drop in Mw and $\mathrm{Mn}$ of $12 \%$ and $23 \%$ in PBS, $22 \%$ and $35 \%$ in acid medium, and $22 \%$ and $31 \%$ in basic medium, respectively. The reason for the drop in molecular weight not correlating with the weight loss is due to the changes in the sample hydrophilicity. Those degraded in basic media became more hydrophilic, enabling the diffusion of the low molecular weight chains, while the samples degraded in PBS or acid medium remained hydrophobous and hindered the diffusion of all molecular chains.

This chain fragmentation influences the thermal properties of the material. The data obtained show an initial rise in crystallinity for the samples degraded in all the media. Similar results have been observed in other studies on the degradation of PLLA membranes $[15,28]$. The increased crystallinity can be attributed to the fact that degradation mainly affects the amorphous phase, producing the reorganization of the 
low molecular-weight chains into new crystallites. These new crystalline regions are less stable than the original crystalline structure and lower the melting temperature.

This chain fragmentation and the morphological defects of the PLA braid affect the mechanical properties of the material, particularly the Young's modulus, breaking load, and elongation. The Young's modulus of the braids after two months of degradation in PBS was reduced by $75 \%$ with respect to the initial value. Samples degraded one month in acid medium lost $70 \%$ and $71 \%$ in basic medium, besides experiencing a considerable increase in the toe zone and increased elongation. The enlarged toe zone can be ascribed to chain fragmentation, which facilitates the deformation of the sample. Previous studies have reported that hydrolytic degradation has a considerable effect on PLA's mechanical properties $[6,11,12,29]$, a finding with which our results agree.

The hydrolytic degradation study provided information on the evolution of the changes in the morphological, thermal and mechanical properties of PLA braid. The results showed no significant changes in either the mass or morphology of the material when degradation is carried out in neutral or acid $\mathrm{pH}$, although there was a significant change in the molecular weights and mechanical properties of the braid. This is an important aspect, as the PLA braid is intended to form part of a tendon or ligament prosthesis. Several studies on braided materials applied to tendon regeneration showed that the material's lack of mechanical properties were replaced by the collagen I and III reinforcement produced by cells [4, 30]. Surrao et al. [31] demonstrated that the application of mechanical stimulation increased extracellular matrix production in fibrous scaffolds. Our hypothesis is that when the prosthesis has been grafted onto damaged tissue, the implant's loss of mechanical properties combined with the effect of mobilization will transfer part of the mechanical loads to the cells. This will trigger the production of extracellular matrix by cells with increased aligned collagen I, which will 
progressively replace the synthetic material with regenerated tissue. In vivo assays will of course have to be performed to confirm the results obtained in vitro.

\section{Conclusions}

This paper describes the effects of hydrolytic degradation on the properties of a PLA hollow braid intended to form part of tendon or ligament prostheses. Three different media were used with neutral, acid and basic $\mathrm{pH}$. The samples immersed in basic medium exhibited a remarkable weight loss as compared to neutral or acid media. The same effect was observed in the morphological changes; the PLA braid degraded in basic medium presented holes and cracks on the surface of the microfibers, while the morphology was almost unaffected in the other media. Molecular weight was found to decrease in all media and was accompanied by a slight increase in crystallinity. All the changes in the material properties affected the mechanical properties of the PLA braid. After two months of degradation the Young's modulus and breaking load were reduced considerably, while the toe zone and elongation were increased. Although in vivo assays will be necessary, from the in vitro results we can conclude that the proposed PLA braid would be good candidate for inclusion in a new prototype of prosthetic tendons or ligaments.

\section{Acknowledgements}

This work has been carried out thanks to the financial support of AITEX (Valencia, Spain). 


\section{References}

1. Garlotta, D., A literature review of poly(lactic acid). Journal of Polymers and the Environment, 2001. 9(2): p. 63-84.

2. Nair, L.S. and C.T. Laurencin, Biodegradable polymers as biomaterials. Progress in Polymer Science, 2007. 32(8-9): p. 762-798.

3. Cooper, J.A., et al., Fiber-based tissue-engineered scaffold for ligament replacement: design considerations and in vitro evaluation. Biomaterials, 2005. 26(13): p. 1523-1532.

4. Ouyang, H.W., et al. The efficacy of bone marrow stromal cell-seeded knitted PLGA fiber scaffold for Achilles tendon repair. in Symposium on Reparative Medicine - Growing Tissues and Organs. 2001. Bethesda, Maryland.

5. Sahoo, S., S.L. Toh, and J.C.H. Goh, A bFGF-releasing silk/PLGA-based biohybrid scaffold for ligament/tendon tissue engineering using mesenchymal progenitor cells. Biomaterials, 2010 31(11): p. 2990-2998.

6. Saha, S.K. and H. Tsuji, Effects of molecular weight and small amounts of $d$ lactide units on hydrolytic degradation of poly(l-lactic acid)s. Polymer Degradation and Stability, 2006. 91(8): p. 1665-1673.

7. Tsuji, H., K. Ikarashi, and N. Fukuda, Poly(l-lactide): XII. Formation, growth, and morphology of crystalline residues as extended-chain crystallites through hydrolysis of poly(l-lactide) films in phosphate-buffered solution. Polymer Degradation and Stability, 2004. 84(3): p. 515-523.

8. Tsuji, H., A. Mizuno, and Y. Ikada, Properties and morphology of poly $(L-$ lactide). III. Effects of initial crystallinity on long-term in vitro hydrolysis of high molecular weight poly(L-lactide) film in phosphate-buffered solution. Journal of Applied Polymer Science, 2000. 77(7): p. 1452-1464.

9. Tsuji, H. and K. Ikarashi, In vitro hydrolysis of poly(L-lactide) crystalline residues as extended-chain crystallites: II. Effects of hydrolysis temperature. Biomacromolecules, 2004. 5(3): p. 1021-1028.

10. Tsuji, H. and Y. Ikada, Properties and morphology of poly(l-lactide) 4. Effects of structural parameters on long-term hydrolysis of poly(l-lactide) in phosphatebuffered solution. Polymer Degradation and Stability, 2000. 67(1): p. 179-189.

11. Yuan, X., A.F.T. Mak, and K. Yao, Comparative observation of accelerated degradation of poly(-lactic acid) fibres in phosphate buffered saline and a dilute alkaline solution. Polymer Degradation and Stability, 2002. 75(1): p. 45-53.

12. Yuan, X., A.F.T. Mak, and K. Yao, Surface degradation of poly(-lactic acid) fibres in a concentrated alkaline solution. Polymer Degradation and Stability, 2003. 79(1): p. 45-52.

13. Tsuji, H., Y. Tezuka, and K. Yamada, Alkaline and enzymatic degradation of Llactide copolymers. II. Crystallized films of poly(L-lactide-co-D-lactide) and poly(L-lactide) with similar crystallinities. Journal of Polymer Science Part B: Polymer Physics, 2005. 43(9): p. 1064-1075.

14. Tsuji, H. and Y. Ikada, Properties and morphology of poly(L-lactide). II. Hydrolysis in alkaline solution. Journal of Polymer Science Part A: Polymer Chemistry, 1998. 36(1): p. 59-66.

15. Cam, D., S.-h. Hyon, and Y. Ikada, Degradation of high molecular weight poly(l-lactide) in alkaline medium. Biomaterials, 1995. 16(11): p. 833-843.

16. Tsuji, H. and K. Nakahara, Poly(L-lactide). IX. Hydrolysis in acid media. Journal of Applied Polymer Science, 2002. 86(1): p. 186-194. 
17. Chung, S., Chain-end scission in acid catalyzed hydrolysis of poly (d,l-lactide) in solution. Journal of Controlled Release, 1995. 34(1): p. 9-15.

18. Kikkawa, Y., et al., Crystallization, stability, and enzymatic degradation of poly(l-lactide) thin film. Biomacromolecules, 2002. 3(2): p. 350-356.

19. Iwata, T. and Y. Doi, Morphology and enzymatic degradation of poly(l-lactic acid) single crystals. Macromolecules, 1998. 31(8): p. 2461-2467.

20. Chu, C.C., Hydrolytic degradation of polyglycolic acid: Tensile strength and crystallinity study. Journal of Applied Polymer Science, 1981. 26(5): p. 17271734.

21. Göpferich, A., Mechanisms of polymer degradation and erosion. Biomaterials, 1996. 17(2): p. 103-114.

22. Vasanthan, N. and O. Ly, Effect of microstructure on hydrolytic degradation studies of poly (l-lactic acid) by FTIR spectroscopy and differential scanning calorimetry. Polymer Degradation and Stability, 2009. 94(9): p. 1364-1372.

23. Maurus, P.B. and C.C. Kaeding, Bioabsorbable implant material review. Operative Techniques in Sports Medicine, 2004. 12(3): p. 158-160.

24. Araque Monrós MC., et al., Procedimiento de obtención de una prótesis biodegradable, A. Universitat Politècnica de València, Editor. 2011: Spain. p. 13.

25. Araque-Monrós MC, G.-M.T., Santos LG, Bernabé SG, Pradas MM, Estellés JM., New concept for a regenerative and resorbable prosthesis for tendon and ligament: Physicochemical and biological characterization of PLA-braided biomaterial. J Biomed Mater Res Part A, 2013. 00A: p. 000-000.

26. Tsuji, H. and Y. Ikada, Properties and morphologies of poly(l-lactide): 1 . Annealing condition effects on properties and morphologies of poly(l-lactide). Polymer, 1995. 36(14): p. 2709-2716.

27. Tsuji, H. and T. Ishida, Poly(L-lactide). X. Enhanced surface hydrophilicity and chain-scission mechanisms of poly(L-lactide) film in enzymatic, alkaline, and phosphate-buffered solutions. Journal of Applied Polymer Science, 2003. 87(10): p. 1628-1633.

28. von Recum, H.A., et al., Degradation of polydispersed poly(l-lactic acid) to modulate lactic acid release. Biomaterials, 1995. 16(6): p. 441-447.

29. Zhang, X., et al., In vitro degradation and biocompatibility of poly(l-lactic acid)/chitosan fiber composites. Polymer, 2007. 48(4): p. 1005-1011.

30. Ouyang HW, et al., Knitted poly-lactide-co-glycolide scaffold loaded with bone marrow stromal cells in repair and regeneration of rabbit Achilles tendon. Tissue Engineering, 2003. 9: p. 431-439.

31. Surrao, D.C., et al., A crimp-like microarchitecture improves tissue production in fibrous ligament scaffolds in response to mechanical stimuli. Acta Biomaterialia, 2012. 8(10): p. 3704-3713. 